\title{
Electrocoagulación con electrodos de aluminio para tratamiento de aguas residuales de curtiembres en Villapinzón, Cundinamarca, Colombia
}

\author{
Electrocoagulation with Aluminum Electrodes for the Wastewater Treatment of Tanneries in \\ Villapinzón, Cundinamarca, Colombia
}

\author{
Miguel Ángel Muñoz Espitia ${ }^{a b}$, Jairo David Cortés Bermúdez ${ }^{a c}$, Rafael Nikolay Agudelo Valencia $^{a d}$ \\ ${ }^{a}$ Programa de Ingeniería Ambiental, Facultad de Ingeniería, Universidad Libre, Colombia \\ ${ }^{\mathrm{b}}$ miguela-munoze@unilibre.edu.co | https://orcid.org/0000-0003-4036-0652 \\ c jairod-cortesb@unilibre.edu.co | https://orcid.org/0000-0003-0530-2553 \\ d rafaeln.agudelov@unilibre.edu.co | https://orcid.org/0000-0002-6646-7725
}

Citation: Muñoz-Espitia, M. A., CortésBermúdez, J. D. y Agudelo-Valencia, R. N. (2022). Electrocoagulación con electrodos de aluminio para tratamiento de aguas residuales de curtiembres en Villapinzón, Cundinamarca, Colombia. Mutis, 12(1). https://doi.org/10.21789/22561498.1783

Recibido: 1 de junio de 2021 Aceptado: 15 de julio de 2021

Copyright: (c) 2021 por los autores. Licenciado para Mutis. Este artículo es un artículo de acceso abierto distribuido bajo los términos y condiciones de la licencia Creative Commons Attribution (https:// https://creativecommons.org/licenses/bync-sa/4.0/).

\section{RESUMEN}

Este articulo proviene de la investigación "Análisis de la eficiencia de la electrocoagulación con electrodos de aluminio para la reducción de turbidez y demanda química de oxígeno (DQO) en las aguas residuales de pelambre en el municipio de Villapinzón, Cundinamarca", la cual se desarrolló en la Universidad Libre, seccional Bogotá, en 2019. Los residuos líquidos provenientes de las curtiembres tienen como problemática común las aguas obtenidas del proceso de pelambre, ya que poseen altas concentraciones de sustancias contaminantes que son vertidas y afectan a cuerpos de agua. Por ello, este estudio tiene como objetivo evaluar un sistema de electrocoagulación a escala de laboratorio para remover turbiedad y DQO, por medio de ensayos realizados en modo discontinuo por períodos de 45 minutos. Con variaciones en la cantidad de electrodos ( 2,4 y 6 ; planos y perforados) y una intensidad de corriente aplicada de $12 \mathrm{~A}$ y $10 \mathrm{~V}$, la máxima remoción de turbiedad y DQO alcanzó valores de 99,77 y $74,18 \%$, respectivamente, cuando se usaron 4 electrodos planos. Para turbiedad, el uso de electrodos perforados permitió alcanzar valores de remoción del $98 \%$ (con 2 electrodos). Esta técnica también permite eliminar sulfuros, empleando 4 y 6 electrodos, alcanzando una remoción de 45,86 y 48,21 \%, respectivamente. En el estudio se pudo determinar que la cantidad adecuada de electrodos para el proceso es 4 . El aumento del número de electrodos reduce la densidad de corriente, aumenta el área de contacto y favorece la disolución de aluminio en exceso.

Palabras clave: electrodos planos, electrodos perforados, turbiedad, DQO, sulfuros

\section{ABSTRACT}

This article derives from the research project "Analysis of the efficiency of electrocoagulation with aluminum electrodes for the reduction of turbidity and chemical oxygen demand (COD) in wastewater from fur cleaning process in the municipality of Villapinzón," developed at Universidad Libre, Bogotá, in 2019. These wastewaters have high concentrations of polluting substances that are discharged and affect effluents. Therefore, this study seeks to evaluate a laboratory-scale electrocoagulation system in order to remove turbidity and COD by means of tests carried out in discontinuous mode for periods of 45 minutes. With variations in the number 
of electrodes (2, 4 and 6; flat and perforated), and an applied current of $12 \mathrm{~A}$ and 10 $\mathrm{V}$, the maximum removal of turbidity, sulfides and COD reached values of 99.77 and $74.1 \%$ respectively, when 4 flat electrodes were used. For turbidity, the use of perforated electrodes allowed obtaining removal values of $98 \%$ (with 2 electrodes). This technique also allows to eliminate sulfides, using 4 and 6 electrodes, thus achieving removal figures of 45.86 and $48.21 \%$, respectively. Findings of this study conclude that the adequate number of electrodes for the process is 4 , and that increasing the number of electrodes reduces the current density, increases the contact area, and favors the dissolution of aluminum excess.

Keywords: Flat electrodes, perforated electrodes, turbidity, COD, sulfides.

\section{INTRODUCCIÓN}

El agua constituye el eje estratégico para el desarrollo económico, social y ambiental, y frente a ello la incesante labor de la ciencia y los avances tecnológicos en la descontaminación de aguas residuales han sido insuficientes, presentándose incluso nuevos objetivos por alcanzar (Muñoz \& Ante, 2017). En el crecimiento exponencial del desarrollo de la industria a nivel mundial el recurso hídrico sufre un notable deterioro, ocasionando problemas sanitarios por la pérdida de propiedades fisicoquímicas, lo que afecta los parámetros óptimos de consumo humano y vida acuática (Llano et al., 2014). Sin embargo, los avances tecnológicos contribuyen en gran medida a la mitigación de la contaminación de cuerpos de agua, con diferentes técnicas en tratamientos previos a sus vertimientos (de-la-Vara-Salazar \& Gutiérrez Pulido, 2008).

La industria de curtiembres transforma la piel cruda y putrescible en un material resistente, no putrescible y de utilidad para la elaboración de diferentes productos de uso frecuente por la sociedad, para lo cual la piel cruda es sometida a una amplia variedad de procesos físicos y químicos que otorgan estabilidad, resistencia y calidad al cuero curtido como producto final (Salwalha et al., 2019). El objetivo fundamental del proceso de curtido es eliminar de la materia prima todos aquellos materiales que no son colágeno, para lo cual se inicia con el remojo de la piel, a fin de hidratarla, y a continuación se realiza el depilado, para retirar el pelo presente en la piel cruda. Posteriormente se realiza el piquelado y curtido del cuero y finalmente el secado y adecuación de la piel, de acuerdo con las necesidades del productor de artículos de cuero. Los procesos que tienen lugar en el curtido de pieles generan abundantes cantidades de agua residual con cargas contaminantes considerables en términos de sólidos, materia orgánica e inorgánica, así como de algunos materiales tóxicos específicos, como sulfuro y cromo (Sengil et al., 2009). Precisamente, el agua residual generada en el proceso de depilado o pelambre es el principal interés de este proyecto, en la medida en que durante esta etapa se generan entre el 60 y el $70 \%$ de las cargas contaminantes presentes en las aguas residuales de una curtiembre (Xu et al., 2013). Estos vertimientos representan un serio problema debido al impacto que pueden tener sobre los cuerpos de agua receptores, pudiendo afectar directamente la concentración de oxígeno disuelto, además de su toxicidad sobre los seres vivos y plantas presentes en el agua.

La presencia de sólidos suspendidos en el agua residual de depilado disminuye la efectividad de las técnicas de oxidación de sulfuros a causa de la selectividad de los agentes oxidantes y del necesario incremento de dosis para lograr una adecuada o correcta remoción previa al vertimiento del agua residual. Con base en lo anterior, es 
recomendable realizar la remoción de sólidos suspendidos en el agua antes de llevar a cabo la oxidación de los sulfuros. La concentración de sólidos suspendidos totales en las aguas residuales del proceso de depilado o pelambre puede variar entre 200 y $1000 \mathrm{mg} / \mathrm{L}$ (Sivagami et al., 2018). La técnica convencional de mayor uso en el tratamiento de dichas aguas es la oxidación catalítica, con aire como fuente de oxígeno y manganeso en el papel de catalizador; posterior a la oxidación se efectúa la clarificación del agua, en donde se hace uso de coagulantes tales como sulfato de aluminio, cloruro férrico, policloruro de aluminio, hidroxicloruro de aluminio y polímeros, para incrementar la densidad de los grumos o floc formados.

Las dosis de coagulantes reportadas para lograr calificaciones razonables varían entre 800 y $900 \mathrm{mg} / \mathrm{L}$ para sulfato de aluminio y cloruro férrico (Haydar \& Aziz, 2009). También se ha observado que el lodo obtenido cuando se hace uso de estos coagulantes generalmente es poroso, poco compacto y con contenidos de agua que varían del 99 al 99,7\%. Con el objeto de reducir o eliminar el uso de agentes coagulantes, en este estudio se evaluará la electrocoagulación con electrodos aluminio para la clarificación de aguas residuales generadas en el proceso de depilado.

La electrocoagulación es un método de tratamiento de aguas que se utiliza principalmente para remover los materiales orgánicos y sólidos suspendidos de varios tipos de efluentes (Maha-Lakshmi \& Sivashanmugan, 2013). Esta técnica hace uso de una fuente de corriente directa y de electrodos, que pueden ser de diferentes metales, tales como hierro, cobre, titanio y aluminio, inmersos en el agua residual. La corriente eléctrica ocasiona la disolución de los ánodos en esta agua, teniendo lugar las diferentes reacciones de oxidación y reducción que permiten descontaminarla. Los cationes liberados en el ánodo contribuyen a reducir la estabilidad de las partículas suspendidas contenidas por causa de la reducción de su potencial zeta (Zongo et al., 2009) y las especies metálicas liberadas en la solución pueden, dependiendo del pH, formar una amplia variedad de especies coaguladas e hidróxidos metálicos que desestabilizan y agregan las partículas suspendidas o se decantan y adsorben contaminantes disueltos (Elabbas et al., 2016). Diversos estudios han demostrado la viabilidad de esta tecnología y su capacidad de eliminación de una amplia gama de contaminantes orgánicos e inorgánicos en varias industrias, incluyendo la textil y del cuero (Sengil et al., 2009).

La electrocoagulación con electrodos de aluminio presenta las siguientes reacciones en los electrodos:

$$
\begin{gathered}
A l \rightarrow \mathrm{Al}^{3+}+3 e^{-} \text {(Ec. 1) } \\
\mathrm{H}_{2} \mathrm{O}+2 e^{-} \rightarrow \mathrm{H}_{2(g)}+2 \mathrm{OH}^{-} \text {(Ec. 2) } \\
\mathrm{Al}^{3+}+3 \mathrm{H}_{2} \mathrm{O} \rightarrow \mathrm{Al}(\mathrm{OH})_{3}+3 \mathrm{H}^{+} \text {(Ec. 3) }
\end{gathered}
$$

La primera reacción ocurre en el ánodo, cuando se aplica corriente eléctri$\mathrm{ca}$, y la segunda reacción en el cátodo. $\mathrm{El} \mathrm{Al}^{3+}$ da lugar en la solución a especies hidrolizadas y monoméricas hasta formar finalmente $\mathrm{Al}(\mathrm{OH})_{3}$, el cual actúa como coagulante principal (Ogando et al., 2018). La electrocoagulación tiene lugar por cuatro mecanismos principales: atrapamiento de contaminantes, adsorción de los mismos, neutralización de cargas y formación de complejos por la reacción del coagulante con los grupos funcionales presentes en los contaminantes 
(Garcia-Segura et al., 2017). Los mecanismos descritos ocurren de manera simultánea y resultan eficientes para remover las impurezas en el agua.

El sector curtidor se caracteriza a nivel mundial y nacional por su gran impacto asociado a la fabricación de calzado. Este hecho ha determinado un aumento en la estructura del mercado del sector a nivel internacional, causando que, a partir del año 2000, los más importantes compradores potenciales de cuero, como Francia e Italia, en la Unión Europea, China, en Asia, y Brasil, en América Latina, aumentaran la producción por parte de fabricantes de productos de cuero, de tal manera que los precios de este y de las pieles incrementaron en forma proporcional. En Colombia, la actividad manufacturera ha sido seleccionada por el Gobierno nacional como uno de los mejores productos prioritarios. El cuero entra a competir en el mercado internacional con el $50 \%$ de la producción nacional, posesionándose como el octavo país en cría de ganado a nivel mundial para suplir las necesidades internacionales (Semana, 2019).

Para el proceso de curtido del cuero es necesario un alto consumo de agua, que llega en la región hasta $100 \mathrm{~m}^{3}$ por tonelada de piel bruta salada, de la cual gran parte se necesita en los procesos de remojo y pelambre, actividades que a su vez generan la mayor carga tóxica. Por ello, se han realizado diversos estudios en el tratamiento de las aguas residuales generadas en tales procesos, con el objetivo de prevenir la contaminación de las fuentes hídricas (Secretaría Distrital de Ambiente, 2019). Por ejemplo, en la Universidad de Nanjing, China, se realizó en 2007 un tratamiento experimental con baja corriente celular de $1 \mathrm{~A}$ y electrodos solubles de acero templado y aluminio, obteniendo como resultado que el acero templado resulta más eficiente, con una mayor remoción por encima del $90 \%$, pero deja una coloración negra típica de sulfuro de hierro (II), mientras que el aluminio elimina en gran proporción el color del efluente, aunque la eficiencia de remoción para las variables de carbono orgánico total (СОT) (amoniaco, sulfuro y turbiedad) fue inferior a $12 \%$. Por consiguiente, para la remoción se trató el agua con electrodos de acero templado, luego se procedió a su filtración y finalmente se trató con electrodos de aluminio. Las tasas de eliminación aumentaron así considerablemente a $68 \%$ de DQO, $43,1 \%$ de amoniaco, $55,1 \%$ de CoT, $96,7 \%$ de sulfuros y $84,3 \%$ de turbiedad (Feng et al., 2007).

Por otra parte, en la Universidad de Sakarya, Turquía, se llevó a cabo en 2009 un experimento de eliminación de DQO, sulfuros y grasas, utilizando corriente continua y examinando $\mathrm{pH}$ inicial, tiempo y densidad de corriente, dando como resultado que al acero templado fue el material más eficiente para la eliminación de estos indicadores, con una eficiencia del $82 \%$ para DQO, $90 \%$ para sulfuros y $96 \%$ para grasas. La densidad óptima de corriente para los dos primeros fue de 35 y 3,5 $\mathrm{mA} / \mathrm{cm}^{2}$ para las grasas; estos resultados se dieron para un tiempo de 10 minutos de electrocoagulación, con un $\mathrm{pH}$ de 3 y un consumo de energía de $5.768 \mathrm{kWh} / \mathrm{m}^{3}$ para DQO, $0,524 \mathrm{kWh} / \mathrm{m}^{3}$ para sulfuros y $0.00015 \mathrm{kWh} / \mathrm{m}^{3}$ para grasas (Sengil et al., 2009). Además, en la ciudad de Estambul, Turquía, Deghles y Kurt investigaron en 2016 acerca de la experimentación híbrida entre electrocoagulación y electrodiálisis para la eliminación de DQO, nitrógeno amoniacal, cromo y color en procesos de curtiembre, analizando los efectos de la densidad de corriente y el tiempo de tratamiento, para optimizar el proceso de uso de electrodos de aluminio y de hierro aplicando electrodiálisis. Los resultados arrojaron que el proceso híbrido era capaz de remover el total de nitrógeno amoniacal y color, además de un $92 \%$ de los DQO; también se observó que la conductividad fue aumentando a medida que se incrementaba el tiempo de tratamiento (Deghles \& Kurt, 2016). 


\section{MATERIALES Y MÉTODOS}

El agua residual para los ensayos fue suministrada por una empresa de curtido de pieles del municipio de Villapinzón, Cundinamarca, y fue tomada de la etapa de depilado o pelambre de las pieles, para posteriormente ser dejada en reposo por un período de 24 horas previo a la realización de los ensayos. En la ejecución de la experimentación, el agua no fue alterada, es decir, no se realizaron ajustes de $\mathrm{pH}$, conductividad, temperatura u otra variable. Se realizó un diseño experimental completamente al azar con un solo factor, dado por el número de electrodos de aluminio utilizados para cada ensayo (2, 4 y 6 electrodos), con el objeto de incrementar el área de contacto de estos con el agua residual y así poder analizar ambos tratamientos: electrocoagulación con electrodos planos (EP) y electrocoagulación con electrodos planos perforados (EPP). Se hizo uso de placas o electrodos planos, así como de electrodos planos con 12 perforaciones de $12,7 \mathrm{~mm}$ de diámetro cada una, para un área total de las perforaciones de $0,0015 \mathrm{~m}^{2}$; debido a que la reducción se realiza en ambas caras del electrodo el área se reduce a 0,003 $\mathrm{m}^{2}$, correspondiente al $10 \%$ del área de cada electrodo. Así, se realizaron dos bloques de tratamientos, con el objeto de determinar inicialmente el efecto del número de electrodos sobre las variables de respuesta seleccionadas, y posteriormente el efecto de las perforaciones. Estas comparaciones se realizaron a partir del análisis de varianza (ANOVA) descrito por de-la-Vara-Salazar y Gutiérrez-Pulido (2008). Las variables de respuesta seleccionadas fueron el porcentaje de remoción de turbiedad y de DQO. Finalmente, se efectuó la comparación entre los resultados promedio de los ensayos que permitieron alcanzar los máximos valores de las variables de respuesta, para lo cual se realizó la prueba de Tukey (de-la-Vara-Salazar \& Gutiérrez Pulido, 2008).

El reactor de electrocoagulación fue conformado por un recipiente plástico de $0,10 \mathrm{~m}$ de ancho, $0,10 \mathrm{~m}$ de largo y $0,20 \mathrm{~m}$ de alto, con un total de 6 ranuras para la ubicación de los electrodos, siendo la distancia entre ranuras de $0,0254 \mathrm{~m}$ y la capacidad del recipiente de $0,002 \mathrm{~m}^{3}$. Cada experimento fue realizado con 0,0015 $\mathrm{m}^{3}$ de agua residual y se empleó una fuente de poder que permitió mantener la intensidad de corriente aplicada a un valor de $12 \mathrm{~A}$ y $10 \mathrm{~V}$ a lo largo de todos los ensayos. Cabe señalar que en cada ensayo la intensidad de corriente y la diferencia de potencial fueron ajustadas para mantener los valores constantes. La agitación en el recipiente de electrocoagulación fue realizada con un agitador magnético a $200 \mathrm{rpm}$. Todos los ensayos fueron realizados en modo discontinuo por un tiempo total de 45 minutos, y cada uno de ellos fue efectuado por triplicado. Después de cada ensayo, el agua residual tratada fue dejada en reposo por un período de 2 horas, y luego cada muestra fue centrifugada para eliminar sólidos remanentes y realizar el análisis de DQO soluble y los demás parámetros considerados. Las determinaciones de $\mathrm{DQO}, \mathrm{pH}$, sulfuros y turbiedad fueron realizadas a partir de los protocolos de análisis indicados en los métodos estándar para análisis de agua potable y residual (APHA et al., 2017).

\section{RESULTADOS}

Las características promedio del agua residual utilizada en los ensayos se presentan en la tabla 1. 
Tabla 1. Características iniciales del agua residual del proceso de depilado

Fuente: elaboración propia.

\begin{tabular}{|c|c|c|}
\hline Variable & Unidad & Valor \\
\hline Temperatura & ${ }^{\circ} \mathrm{C}$ & 19,5 \\
\hline $\mathrm{pH}$ & & 12,3 \\
\hline Turbiedad & UNT & 4,164 \\
\hline DQO & $\mathrm{mg} \mathrm{O}_{2} / \mathrm{L}$ & 4,450 \\
\hline Sulfuros & $\mathrm{mg} / \mathrm{L}$ & 1,450 \\
\hline
\end{tabular}

Los resultados de las variables de respuesta para cada ensayo realizado se presentan en la tabla 2. A simple vista, se puede notar que el número de electrodos sí es relevante para lograr mayores valores de porcentaje de remoción de turbiedad y DQo.

Tabla 2. Valores del porcentaje de remoción de turbiedad y DQo para los distintos ensayos realizados

\begin{tabular}{|c|c|c|c|}
\hline $\begin{array}{l}\text { Número } \\
\text { de EP }\end{array}$ & $\begin{array}{c}\% \text { de remoción de } \\
\text { turbiedad }\end{array}$ & $\begin{array}{l}\text { \% de remoción de } \\
\text { DQO }\end{array}$ & $\begin{array}{l}\text { \% remoción de } \\
\text { sulfuros }\end{array}$ \\
\hline 2 & 75,62 & 16,63 & 11,86 \\
\hline 2 & 75,50 & 16,85 & 10,97 \\
\hline 2 & 75,74 & 16,40 & 11,10 \\
\hline 4 & 99,86 & 74,16 & 34,97 \\
\hline 4 & 99,76 & 74,72 & 34,07 \\
\hline 4 & 99,69 & 73,71 & 34,76 \\
\hline 6 & 98,90 & 31,46 & 26,07 \\
\hline 6 & 98,50 & 31,69 & 26,69 \\
\hline 6 & 98,35 & 31,91 & 25,66 \\
\hline $\begin{array}{l}\text { Número de } \\
\text { EPP }\end{array}$ & $\begin{array}{c}\% \text { de remoción de } \\
\text { turbiedad }\end{array}$ & $\begin{array}{l}\text { \% de remoción de } \\
\text { DQO }\end{array}$ & $\begin{array}{l}\text { \% remoción de } \\
\text { sulfuros }\end{array}$ \\
\hline 2 & 98,25 & 21,80 & 19,52 \\
\hline 2 & 98,07 & 21,12 & 19,45 \\
\hline 2 & 98,54 & 21,35 & 20,14 \\
\hline 4 & 99,63 & 52,58 & 45,86 \\
\hline 4 & 99,52 & 51,69 & 46,14 \\
\hline 4 & 99,75 & 52,81 & 45,59 \\
\hline 6 & 99,15 & 57,30 & 48,41 \\
\hline 6 & 99,44 & 58,43 & 47,93 \\
\hline 6 & 99,40 & 57,30 & 48,28 \\
\hline
\end{tabular}

Fuente: elaboración propia. 
Los datos reportados en la tabla 2 señalan que los mayores porcentajes de remoción para las variables de respuesta seleccionadas se logran con 4 y 6 electrodos, observación que se hace más notoria para el porcentaje de remoción de turbiedad con EP. Por otro lado, no se aprecia una diferencia significativa entre el uso de 4 y 6 electrodos. En el caso del porcentaje de remoción de turbiedad se observan diferencias significativas al variar el número de electrodos, mientras que para los EPP este porcentaje es muy similar en todos los ensayos, pero la remoción de DQO varía al aumentar el número de electrodos. De la misma manera ocurre con la remoción de sulfuros, al incrementar el número de electrodos se aumenta la remoción de estos y también se aprecia mayor remoción al hacer uso de EPP. Estos resultados se exponen en la tabla 3.

Tabla 3. Valores máximos de remoción ( $\mathrm{N}$ = número de electrodos empleados en el ensayo)

\begin{tabular}{|c|c|c|c|c|c|c|}
\hline \multirow{2}{*}{ Tipo de electrodo } & \multicolumn{7}{|c|}{ \% de remoción } \\
\cline { 2 - 7 } & $\mathbf{N}$ & Turbiedad & $\mathbf{N}$ & DQO & $\mathbf{N}$ & Sulfuros \\
\hline EP & 4 & 99,77 & 4 & 74,20 & 4 & 34.60 \\
\hline EPP & 4 & 99,63 & 6 & 57,68 & 6 & 48.21 \\
\hline
\end{tabular}

Fuente: elaboración propia.

Los resultados obtenidos indican que los valores máximos en la remoción de cada uno de los parámetros analizados fue 99,77 y $74,20 \%$, con 4 EP, para turbiedad y DQO, respectivamente, y de 48,21\%, con 6 EPP, para sulfuros. Se puede apreciar que en términos de remoción de turbiedad el número de electrodos y su diseño es poco significativo, pero que el uso de 4 EP permite alcanzar mayor remoción de DQO en comparación con los EPP. No obstante, la remoción de sulfuros es mayor cuando se hace uso de 6 EPP que cuando se hace uso de $6 \mathrm{EP}$, alcanzando una diferencia cercana a $14 \%$. La reducción de turbiedad se presenta principalmente por la oxidación del aluminio del que está hecho el ánodo, el cual posteriormente, dado el elevado valor de $\mathrm{pH}$ del agua residual, favorece la desestabilización de las partículas y su precipitación por la formación de hidróxido de aluminio. En cuanto a la remoción de sulfuros, la diferencia puede deberse a la reducción de área y, por lo tanto, la mayor densidad de corriente y convección en el sistema reactivo. El análisis estadístico de los resultados se realizó por medio de ANOVA y es presentado en la tabla 4. 
Tabla 4. ANOVA de un solo factor para las variables de respuesta analizadas: (a) \% de remoción de turbiedad con EP, (b) porcentaje de remoción de DQO con EP, (c) porcentaje de remoción de turbiedad con EPP, y (d) porcentaje de remoción de DQO con EPP

\begin{tabular}{|c|c|c|c|c|c|c|c|c|c|c|c|}
\hline \multicolumn{6}{|c|}{ ANOVA turbiedad EP } & \multicolumn{6}{|c|}{ ANOVA DQO EP } \\
\hline Fuente & $\mathrm{GL}$ & sc Ajust. & $\begin{array}{c}\text { MC } \\
\text { Ajust. }\end{array}$ & Valor F & Valor $\mathrm{p}$ & Fuente & GL & sc Ajust. & MC Ajust. & Valor F & $\begin{array}{c}\text { Valor } \\
\mathrm{p}\end{array}$ \\
\hline $\begin{array}{l}\text { Número } \\
\text { de placas }\end{array}$ & 2 & $1.111,47$ & 555,737 & $16.137,97$ & 0,000 & $\begin{array}{l}\text { Número } \\
\text { de placas }\end{array}$ & 2 & $5.347,53$ & $2.673,76$ & $22.424,69$ & 0,000 \\
\hline Error & 6 & 0,21 & 0,034 & & & Error & 6 & 0,72 & 0,12 & & \\
\hline Total & 8 & $1.111,68$ & & & & Total & 8 & $5.348,24$ & & & \\
\hline \multicolumn{6}{|c|}{ Resumen del modelo } & \multicolumn{6}{|c|}{ Resumen del modelo } \\
\hline \multirow{2}{*}{$S$} & \multirow{2}{*}{ R-cuad. } & R-cuad. & R-cuad. & & & & & (ajustado) & (pred) & & \\
\hline & & (ajustado) & (pred) & & & 0,345301 & $99,99 \%$ & $99,98 \%$ & $99,97 \%$ & & \\
\hline 0.185571 & $99,98 \%$ & $99,98 \%$ & $99,96 \%$ & & & & & & & & \\
\hline \multicolumn{6}{|c|}{ (a) } & \multicolumn{6}{|c|}{ (b) } \\
\hline \multicolumn{6}{|c|}{ ANOVA turbiedad EPP } & \multicolumn{6}{|c|}{ ANOVA DQO EPP } \\
\hline Fuente & $\mathrm{GL}$ & sc Ajust. & $\begin{array}{c}\mathrm{MC} \\
\text { Ajust. }\end{array}$ & Valor F & Valor $\mathrm{p}$ & Fuente & $\mathrm{GL}$ & sc Ajust. & MC Ajust. & Valor F & $\begin{array}{c}\text { Valor } \\
\mathrm{p}\end{array}$ \\
\hline $\begin{array}{c}\text { Número } \\
\text { de placas } \\
\text { PP }\end{array}$ & 2 & 29.982 & 149.911 & 48,12 & 0,000 & $\begin{array}{c}\text { Número } \\
\text { de placas } \\
\text { PP }\end{array}$ & 2 & $2.282,98$ & $1.141,49$ & $4.413,18$ & 0,000 \\
\hline Error & 6 & 0,1869 & 0,03115 & & & Error & 6 & 1,55 & 0.26 & & \\
\hline Total & 8 & 31,851 & & & & Total & 8 & $2.284,53$ & & & \\
\hline \multicolumn{6}{|c|}{ Resumen del modelo } & \multicolumn{6}{|c|}{ Resumen del modelo } \\
\hline \multirow{2}{*}{ S } & \multirow{2}{*}{ R-cuad. } & R-cuad. & R-cuad. & & & \multirow{2}{*}{$\mathrm{s}$} & \multirow{2}{*}{ R-cuad. } & R-cuad. & R-cuad. & & \\
\hline & & (ajustado) & (pred) & & & & & (ajustado) & (pred) & & \\
\hline 0,176503 & $94,13 \%$ & $92,18 \%$ & $86,80 \%$ & & & 0,508581 & $99,93 \%$ & $99,91 \%$ & $99,85 \%$ & & \\
\hline \multicolumn{6}{|c|}{ (c) } & \multicolumn{6}{|c|}{ (d) } \\
\hline
\end{tabular}

Fuente: elaboración propia.

El ANOVA indica que el número de electrodos que se emplearon en los ensayos de electrocoagulación afecta los valores obtenidos de las variables de respuesta, sin importar el tipo de electrodos. Por medio de la prueba de Tukey se determinó que existen diferencias significativas entre los resultados promedio de las variables de respuesta por efecto de los tipos de electrodos (tabla 5).

Tabla 5. Resultados del análisis de Tukey.

\begin{tabular}{|c|c|c|c|c|c|c|c|c|c|}
\hline Diferencia de Tipo de electrodos niveles & $\begin{array}{r}\text { Diferencia } \\
\text { de medias } \\
\end{array}$ & $\begin{array}{r}\text { EE de } \\
\text { diferencia } \\
\end{array}$ & $\begin{array}{l}\text { IC simultáneo } \\
\text { de } 95 \%\end{array}$ & Valor $\mathrm{T}$ & Diferencia de Tipo de electrodos niveles & $\begin{array}{r}\text { Diferencia } \\
\text { de medias }\end{array}$ & $\begin{array}{r}\text { EE de } \\
\text { diferencia } \\
\end{array}$ & $\begin{array}{c}\text { IC } \\
\text { simultáneo } \\
\text { de 95\% }\end{array}$ & Valor T \\
\hline Electrodos planos - Electrodos perforados & -7.76 & 2.82 & $(-13.80,-1.71)$ & -2.75 & Electrodos planos - Electrodos perforados & -2.87 & 5.27 & $(-14.18,8.43)$ & -0.55 \\
\hline Diferencia de Tipo de electrodos niveles & $\begin{array}{r}\text { Valor p } \\
\text { ajustado } \\
\end{array}$ & & & & Diferencia de Tipo de electrodos niveles & $\begin{array}{r}\text { Valor p } \\
\text { ajustado } \\
\end{array}$ & & & \\
\hline Electrodos planos - Electrodos perforados & 0.016 & & & & Electrodos planos - Electrodos perforados & 0.594 & & & \\
\hline
\end{tabular}

Fuente: elaboración propia.

Con base en la prueba de Tukey, se determinó que en cuanto a remoción de turbiedad existen diferencias significativas entre hacer uso de EP O EPP (tabla 5a), mientras que con respecto al porcentaje de remoción de DQO, no existen diferencias significativas en los resultados por causa del tipo de electrodos (tabla 5b). La figura 1 permite observar los gráficos de efectos principales para cada variable de respuesta 
con respecto al número y al tipo de electrodos.

Figura 1. Número de placas contra porcentaje de remoción de turbiedad y DQO

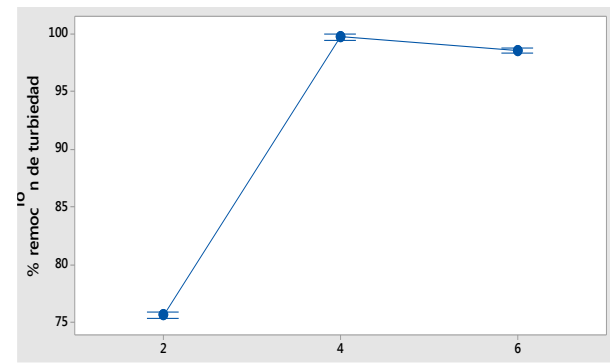

(a)

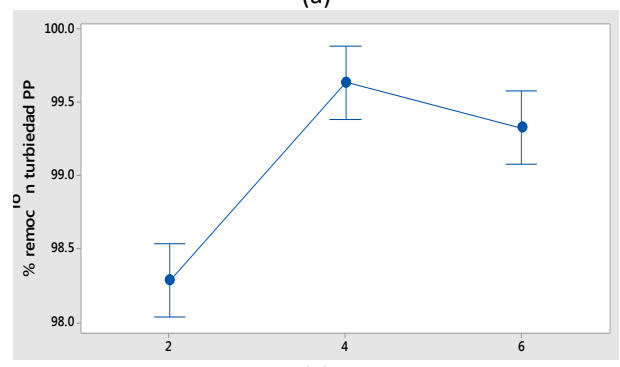

(c)

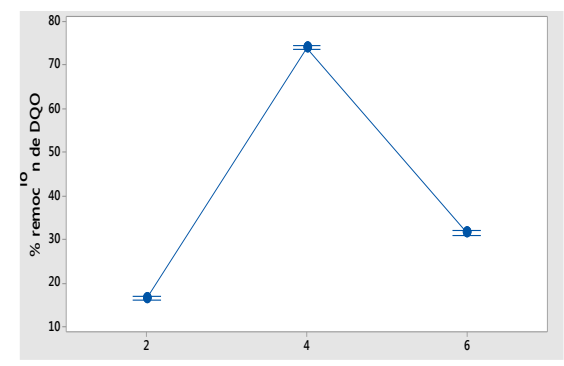

(b)

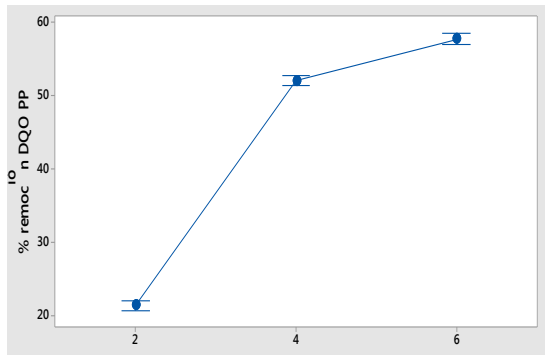

(d)

(a) porcentaje de remoción de turbiedad (EP), (b) porcentaje de remoción de DQO (EP), (c) porcentaje de remoción de turbiedad (EPP), y (d) porcentaje de remoción de DQO (EPP).

Fuente: elaboración propia.

En la figura 1a se observa que el número de electrodos o placas de aluminio utilizadas en el proceso de electrocoagulación influye en la remoción de turbiedad, notándose la diferencia principalmente entre el uso de $2(75,62 \%)$ y 4 electrodos $(99,7 \%)$, en tanto que cuando se pasa de 4 a 6 electrodos se observa una leve disminución del porcentaje de remoción (98,58\%). Esta diferencia se puede considerar poco significativa, de manera que para más de 4 electrodos no se presentan cambios significativos en la remoción de turbiedad. La situación es similar cuando los electrodos son perforados (figura 1c), en cuyo caso la diferencia entre porcentajes de remoción no es significativa y en promedio alcanzó un valor de $99,08 \%$ cuando se hace uso de 4 y 6 electrodos. Con relación al DQo, al hacer uso de EP (figura 1 b), la remoción aumenta, al pasar de $2(16,63 \%)$ a 4 EP $(74,19 \%)$, pero disminuye cuando se emplean 6 EP (31.69\%), situación que puede deberse a la reducción de la densidad de corriente en el sistema.

Por otra parte, cuando los electrodos de aluminio son perforados (figuras $1 \mathrm{c}$ y $1 d$ ) resulta notable que el uso de 4 electrodos permite alcanzar la mayor remoción de turbiedad y DQO, además de que las barras de error en el gráfico evidencian que las variables de respuesta no presentan diferencias significativas cuando se hace uso de 4 y 6 electrodos. La remoción de turbiedad en todos los ensayos se explica por la disminución del potencial zeta de las partículas presentes en el agua, lo que hace que tiendan a aglomerarse (Garcia-Segura et al., 2017). De otro lado, los hidróxidos metálicos e hidróxidos orgánicos insolubles formados durante la electrocoagulación propician la remoción de sólidos suspendidos y, en consecuencia, la reducción de turbiedad. Los ensayos adicionales realizados para analizar la remoción de sulfuros indican que el mayor valor de remoción se logra cuando se hace uso de EPP, con un valor que varía 
entre $45,86 \%$ cuando se emplean 4 electrodos y $48,21 \%$ cuando se utilizan 6 ; esto puede ser nuevamente atribuido al aumento de la densidad de corriente por causa de las perforaciones, así como a la convección en el sistema reactivo. La figura 2 muestra los rendimientos de la prueba de Tukey sobre los resultados de los ensayos efectuados, a partir de lo cual se determina si existen diferencias significativas en los valores medios de remoción obtenidos para los dos distintos tratamientos realizados (Khemis et al., 2005).

Figura 2. Gráficas de la prueba de Tukey para determinar diferencias entre promedios de porcentaje de remoción.

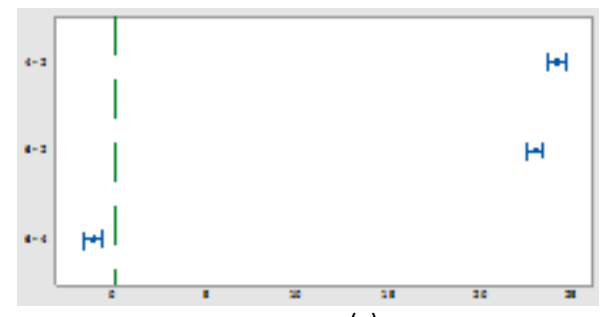

(a)

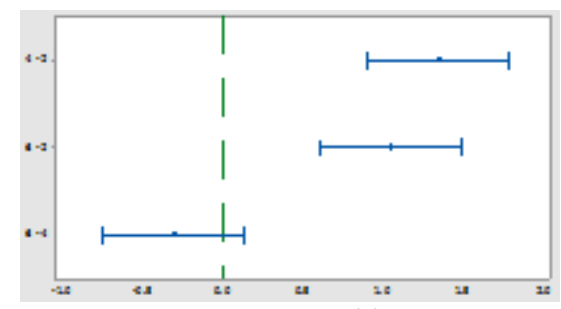

(c)

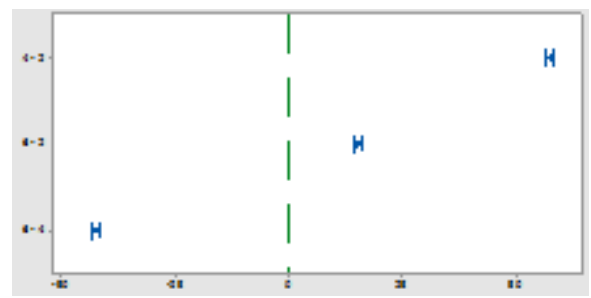

(b)

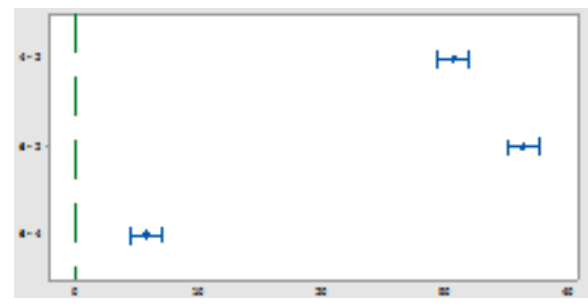

(d)

(a) porcentaje de remoción de turbiedad con EP, (b) porcentaje de remoción de DQO con EP, (c) porcentaje de remoción de turbiedad con EPP y (d) porcentaje de remoción de DQO con EPP.

Fuente: elaboración propia.

Los intervalos en la figura 2 corresponden a la prueba de Tukey para un nivel de confianza de $95 \%$. A partir de estos intervalos se determina que en todos los ensayos realizados con EP (figuras $2 \mathrm{a}$ y $2 \mathrm{~b}$ ) los resultados promedio difieren entre sí. Por su parte, cuando se hace uso de EPP (figuras $2 \mathrm{c}$ y $2 \mathrm{~d}$ ) existen diferencias significativas en los resultados promedio cuando se pasa de 4 a 6 electrodos, excepto para remoción de turbiedad, en donde no se presentan tales diferencias significativas entre resultados. Con relación a la pérdida por oxidación de aluminio en el ánodo, la masa de aluminio que se disuelve se calculó utilizando la ley de Faraday (Benhagji et al., 2011):

$$
m=\frac{I * t * P M}{z * F} \quad(\text { Ec. } 4)
$$

En la ecuación anterior, $m$ es la masa de aluminio disuelto ( $g$ ), I es la intensidad de corriente aplicada (A), $t$ el tiempo de reacción (s), $P M$ la masa molecular del aluminio, $z$ el número de electrones implicados en la reacción y $F$ la constante de Faraday $\left(96.485,34 \mathrm{~A} /\left(\mathrm{mol}^{*} \mathrm{~s}\right)\right)$. De acuerdo con lo anterior, la masa teórica de aluminio disuelto en cada ensayo fue de $3.019 \mathrm{~g}$, equivalente a una concentración de $1.509,99 \mathrm{mg} / \mathrm{L}$ de aluminio en solución. La remoción de sulfuros en este tipo de agua 
residual también se debe a la formación de sulfuro de aluminio $\left(\mathrm{Al}_{2} \mathrm{~S}_{3}\right)$ (Moradi \& Moussavi, 2018), además de las reacciones de oxidación que ocurren en los electrodos. Una variable que se debe considerar en los procesos de electrocoagulación es la densidad de corriente aplicada, la cual se puede calcular a partir de la siguiente ecuación:

$$
\rho_{I}=\frac{\text { Intensidad de corriente }(A)}{\text { Área de los electrodos en contacto con el agua residual }\left(m^{2}\right)}
$$

De acuerdo con lo obtenido, al hacer uso de 4 electrodos, ya sea EP O EPP, se alcanzan mejores resultados de remoción de las variables analizadas, cuando la densidad de corriente $\rho$ tiene un valor de $75 \frac{\mathrm{A}}{\mathrm{m}^{2}}$ para EP y $81.17 \frac{\mathrm{A}}{\mathrm{m}^{2}}$ para EPP; estos valores de densidad de corriente se encuentran en el rango reportado por otras investigaciones en el área del tratamiento de aguas residuales de curtiembres (Keerthi et al., 2013; Murugananthan et al., 2004; Papadopoulos et al., 2019).

\section{DISCUSIÓN}

En Colombia, la normatividad en términos de vertimiento de aguas residuales a cuerpos de agua superficiales está reglamentada en la Resolución 631 de 2015. Para el caso específico de las curtiembres ubicadas en la cuenca del río Bogotá, la Corporación Autónoma Regional de Cundinamarca (CAR), a través del Acuerdo 46 de 2006, plantea los objetivos de calidad del agua para el río, a partir de los cuales se determina la norma de vertimientos para el sector curtidor. Las máximas concentraciones de DQO, sulfuros y sólidos suspendidos totales (SST) en un vertimiento de este tipo de agua residual debe ser de $120 \mathrm{mg} \mathrm{O}_{2} / \mathrm{L}$ para DQO, $1 \mathrm{mg} / \mathrm{L}$ para sulfuros y $50 \mathrm{mg} / \mathrm{L}$ para SST. Los valores obtenidos de remoción en el presente proyecto fueron de $99,7,74,20$ y $48,21 \%$ de turbiedad, DQO y sulfuros, respectivamente. A partir de las condiciones iniciales del agua se determina que después del proceso realizado la turbiedad se reduce hasta $9,58 \mathrm{NTU}$, los DQO se disminuyen a $1.148,25 \mathrm{mg} \mathrm{O} / \mathrm{L}$ y los sulfuros caen a $751 \mathrm{mg} / \mathrm{L}$, resultados que sobrepasan los valores establecidos por la normatividad que rige al sector curtidor asentado en la cuenca del río Bogotá, aunque el avance es significativo, toda vez que se logra clarificar el agua sin la necesidad de reducir el pH y adicionar insumos químicos, lo que permite posteriormente pasar a la reducción de DQO y sulfuros remanentes en el agua por medio de otras técnicas de tratamiento de aguas o por la intensificación de la técnica analizada.

El uso de EPP favorece la convección en el sistema y por lo tanto el contacto continuo del agua residual con la superficie de los electrodos, además de incrementar la densidad de corriente por efecto de la reducción de área causada por la presencia de perforaciones en los electrodos. En términos de reducción de turbiedad, el uso de 4 electrodos y la respectiva densidad de corriente aplicada resulta ser la configuración adecuada para alcanzar el mayor porcentaje de remoción, cercana al $100 \%$, de tal manera que incrementar el número de electrodos no representa un aumento significativo de remoción.

Las pruebas estadísticas realizadas indican que no existe diferencia significativa entre el uso de EP O EPP para remover turbiedad. En el caso de DQO, los EP dan lugar a una remoción un poco mayor, lo cual se puede explicar como consecuencia de la mayor área de contacto que cuando se emplean EPP. La remoción de sulfuros no fue significativa desde el punto de vista normativo, pero a nivel operativo el resultado fue de $48 \%$ para EPP, lo que hace posible reducir el porcentaje restante por otra técnica 
de tratamiento. La normatividad no presenta valores máximos permisibles para turbiedad, pero aproximadamente, para valores menores que 100 UNT, se puede equiparar esta variable con el valor de SST, de manera que la técnica estudiada permite cumplir con el valor normativo para SST.

\section{CONCLUSIONES}

El número y tipo de electrodos influye sobre el porcentaje de remoción de turbiedad y DQO en las condiciones experimentales analizadas. El uso de 4 EP (y por lo tanto de una $\rho_{l}=75 \mathrm{~A} / \mathrm{m}^{2}$ ) es la condición operativa para la cual se logran los mayores resultados de remoción de turbiedad, DQO y sulfuros $(99,77,74,20$ y $34,6 \%$, respectivamente). Cuando se hace uso de 6 EPP y la densidad de corriente es $\rho_{l}=81,17 \mathrm{~A} / \mathrm{m}^{2}$, se logra remover $99,33 \%$ de turbiedad, $57,68 \%$ de DQO y $48,21 \%$ de sulfuros. La electrocoagulación es una técnica fácil de operar y controlar, lo cual representa un beneficio para quienes apliquen este tipo de tratamiento. Las diferencias de área y régimen de mezcla entre EP y EPP resultan significativas para considerar el número de electrodos que se deben utilizar en el proceso. Desde el punto de vista operativo, el proceso analizado presenta el beneficio de clarificar el agua sin la adición de insumos químicos adicionales, además de disminuir las concentraciones de DQO y sulfuros, hecho que permite reducir esfuerzos en tratamientos posteriores para alcanzar las metas de vertimiento establecidas por la normatividad ambiental para el sector curtidor ubicado en la cuenca del río Bogotá. El proceso puede ser igualmente intensificado por técnicas de oxidación avanzada para alcanzar las metas normativas de DQO y sulfuros.

\section{REFERENCIAS}

American Public Health Association [APHA], American Water Works Association [AWWA], \& Water Environment Federation [WEF]. (2017). Standard methods for the examination of water and wastewater. APHA.

Benhagji, A., Taleb-Ahmed, M., \& Maachi, R. (2011). Electrocoagulation and effects of cathode materials on the removal of pollutants from tannery wastewater of Rouïba. Desalination, 277(1-3), 128-134. https://doi.org/10.1016/j.desal.2011.04.014

De-la-Vara-Salazar, R., \& Gutiérrez-Pulido, H. (2008). Análisis y diseño de experimentos. McGraw-Hill.

Deghles, A., \& Kurt, U. (2016). Traetment of tannery wasterwater by hibrid electrocoagulation/electrodyalisis process. Chemical Engineering and Processing: Process Intensification, 104, 43-50. https://doi.org/10.1016/j.cep.2016.02.009

Elabbas, S., Ouazzani, N., Mandi, L., Berrekhis, F., Perdicakis, M., Pontvianne, S., Pons, M. N., Lapicque, F., \& Leclerc, J. P. (2016). Treatment of highly concentrated tannery wastewater using electrocoagulation: Influence of the quality of aluminium used for the electrode. Journal of Hazardous Materials, 319, 69-77. https://doi.org/10.1016/j.jhazmat.2015.12.067

Feng, J. W., Sun, Y. B., Zheng, Z., Zhang, J. B., Li, S., \& Tian, Y. (2007). Treatment of tannery wasterwater by electrocoagulation. Journal of Enviromental Sciences, 19(12), 1409-1415. https://doi.org/10.1016/S1001-0742(07)60230-7 
Garcia-Segura, S., Eiband, M. M., de-Melo, J. V., \& Martinez-Huitle, C. A. (2017). Electrocoagulation and advanced electrocoagulation processes: A general review about the fundamentals, emerging applications and its association with other technologies. Journal of Electroanalytical Chemistry, 801, 267-299. https://doi.org/10.1016/j.jelechem.2017.07.047

Haydar, S., \& Aziz, J. A. (2009). Coagulation-floculation studies of tannery wastewater using combination of alum with cationic and anionic polymers. Journal of Hazardous Materials, 168(2-3), 1035-1040. https://doi.org/10.1016/j.jhazmat.2009.02.140

Keerthi, P., Suganthi, V., Mahalakshmi, M., \& Balasubramanian, N. (2013). Development of hybrid membrane bioreactor for tannery effluent treatment. Desalination, 309, 231-236. https://doi.org/10.1016/j.desal.2012.10.014

Khemis, M., Tanguy, G., Leclerc, P., Valentin, G., \& Lapicque, F. (2005). Electrocagulation for the treatment of oil suspensions: Relation between the rates of electrode reactions and the efficiency of waste removal. Process Safety and Environmental Protection, 83(1), 50-57. https://doi.org/10.1205/psep.03381

Llano, B. A., Cardona, J. F., Ocampo, D., \& Ríos, A. (2014). Tratamiento fisicoquímico de las aguas residuales generadas en el proceso de beneficio de arcillas y alternativas de uso de los lodos generados en el proceso. Información Tecnológica, 25(3), 73-82. http://dx.doi.org/10.4067/S0718-07642014000300010

Maha-Lakshmi, P., \& Sivashanmugan, P. (2013). Treatment of oil tanning effluent by electrocoagulation: Influence of ultrasound and hybrid electrode on COD removal. Separation and Purification Technology, 116, 378-384. https://doi.org/10.1016/j.seppur.2013.05.026

Moradi, M., \& Moussavi, G. (2018). Enhanced treatment of tannery wastewater using the electrocoagulation process combined with UVC/VUV photoreactor: Parametric and mechanistic evaluation. Chemical Engineering Journal, 358, 1038-1046. https://doi.org/10.1016/j.cej.2018.10.069

Muñoz, D., \& Ante, L. (2017). Estandarización de un protocolo in vitro para el control biológico de Eichhornia crassipes con Neochetina. Biotecnología en el Sector Agropecuario y Agroindustrial, 15, 40-48. https://doi.org/10.18684/BSAA(15)40-48

Murugananthan, M., Bhaskar-Raju, G., \& Prabhakar, S. (2004). Removal of sulfide, sulfate and sulfite ions by elctro coagulation. Journal of Hazardous Materials, 109(1-3), 37-44. https://doi.org/10.1016/j.jhazmat.2003.12.009

Ogando, F. B., de-Aguiar, C. L., Viotto, J. N., Heredia, F. J., \& Hernanz, D. (2018). Removal of phenolic, turbidity and color in sugarcane juice by electrocagulation as a sulfur-free process. Food Research International, 122, 643-652. https://doi.org/10.1016/j.foodres.2019.01.039

Papadopoulos, K. P., Argyriou, R., Economou, C. N., Charalampous, N., Dailianis, S., Tatoulis, T. I., Tekerlekopoulou, A. G., \& Vayenas, D. V. (2019). Treatment of printing ink wastewater using electrocoagulation. Journal of Environmental Management, 237, 442-448. https://doi.org/10.1016/j.jenvman.2019.02.080 
Salwalha, H., Alsharabaty, R., Sarsour, S., \& Al-Jabari, M. (2019). Wastewater from leather tanning and processing in Palestine: characterization and management aspects. Journal of Enviromental Management, 251. https://doi.org/10.1016/j.jenvman.2019.109596

Secretaría Distrital de Ambiente. (2019, septiembre 4). Guia de producción más limpia para el sector de curtiembres de Bogotá enfoque en vertimientos y residuos.

http://www.ambientebogota.gov.co/documents/24732/3987253/Gu\%C3\%ADa+de+p roducci\%C3\%B3n+m\%C3\%A1s+limpia+para+el+sector+curtiembres+de+Bogot\%C3\%A 1.+Enfoque+en+vertimientos+y+residuos.pdf

Semana. (2019, septiembre 4). Semana Industrial del Cuero. https://www.semana.com/especiales/articulo/industria-del-cuero/6760-3/

Sengil, I. A., Kulac, S., \& Özacar, M. (2009). Treatment of tannery liming drum wastewater by electrocoagulation. Journal of Hazardous Materials, 167(1-3), 940-946. https://doi.org/10.1016/j.jhazmat.2009.01.099

Sivagami, K., Sakthivel, K. P., \& Nambi, I. M. (2018). Advance oxidation processes for the treatment of tannery wastewater. Journal of Environmental Chemical Engineering, 6(3), 3656-3663. https://doi.org/10.1016/j.jece.2017.06.004

Xu, W., Zhang, J., \& Hao, L. (2013). Hair-saving enzyme-assisted unhairing: Effects of sodium hydrosulfide and peroxide. 32nd Congress of the International Union of Leather Technologists and Chemist Societies, IULTCS 2013. http://www.researchgate.net/publication/289191170_Hair-saving_enzymeassited_unhairing_Effects_of_sodium_hydrosulfide_and_peroxide

Zongo, I., Maiga, A., Wethe, J., Valentin, G., Leclerc, J. P., Paternotte, G., \& Lapicque, F. (2009). Electrocoagulation for the treatment of textile wastewaters with Al or Fe electrodes: Compared variations of COD levels, turbidity and absorbance. Journal of Hazardous Materials, 169, 70-76. https://doi.org/10.1016/j.jhazmat.2009.03.072 